\title{
The irrelevance of adhesive platelet estimations after thrombosis
}

\author{
R. D. EASTHAM \\ From the Department of Pathology, Frenchay Hospital, Bristol
}

SYNOPSIS It has been found that adhesive platelet counts are not of clinical use either after $\stackrel{\circ}{\rightarrow}$ surgical operation or during long-term anticoagulant therapy in the detection of thrombosis. $\vec{\subseteq}$ Calculation of the theoretical risk of development of platelet thrombi related to the platelet count suggests a possible reason for this absence of clinical significance. The evidence that $\vec{\theta}$ platelet adhesiveness alters because of uncontrollable platelet variables and is controlled at. least in part by plasma factors is discussed. The possibility of measuring the plasma factors is considered briefly.

Estimation of platelet adhesiveness has not been found to be clinically useful either during longterm anticoagulant therapy (Eastham, 1966; Eastham and Avis, 1966) or in the detection of venous thrombosis after surgery (Negus, Pinto, and Brown, 1969). A reassessment of this absence of obvious clinical significance has now been attempted, as there is evidence that the degree of platelet adhesiveness can change rapidly without any variation in the total platelet count, and because it now appears that platelet adhesiveness is greatly influenced by plasma factors (Bennett, 1968; Besterman, Myat, and Travadia, 1967; Bolton, Hampton, and Mitchell, 1967).

\section{Methods and Materials}

Total platelet counts and adenosine diphosphate (ADP)-induced adhesive platelet counts were performed on a total of 650 blood samples from six male volunteers and from 57 patients during long-term anticoagulant therapy over a period of six to 24 months, using the methods of Eastham (1963 and 1964). The patients studied included two men with mitral valve stenosis and attacks of embolısm, eight men and one woman with Received for publication 8 October 1969. deep vein thrombosis and a history of attacks of pulmonary embolism, and 45 men and one woman who had suffered from myocardial infarction. All these patients were being treated with oral anticoagulants.

In addition, total and adhesive platelet counts were obtained daily from a 24-year-old woman $\delta$ suffering from idiopathlc thrombocytopenic purpura, who underwent spienectomy to relieve? this condition.

\section{Results}

The mean adhesive platelet count for the whole group was $56 \%(1 \mathrm{SD}=8.8 \%)$ of the total 0 platelet count. Mean values for the adhesive $\overparen{\Phi}$ platelet counts in the six volunteers not taking $\stackrel{\mathscr{P}}{+}$ oral anticoagulants $(54.5 \%)$, nine patients with 0 venous thromboses $(55.2 \%)$, and 46 patients following myocardial infarction $(56.2 \%)$ were not $\stackrel{D}{\circ}$ significantly different from the total mean value. $\stackrel{\mathbb{D}}{\circ}$ Pegrum, Shaw, and Wolff (1967), using the same 0 method, obtained a mean value of $60.5 \pm 6.35 \%$ from 67 normal control subjects. This result is $\frac{0}{0}$ significantly different from the mean value for these patients on oral anticoagulants $(P<0.01)$. $\frac{\bar{\partial}}{\partial}$ There is a direct correlation between the mean 


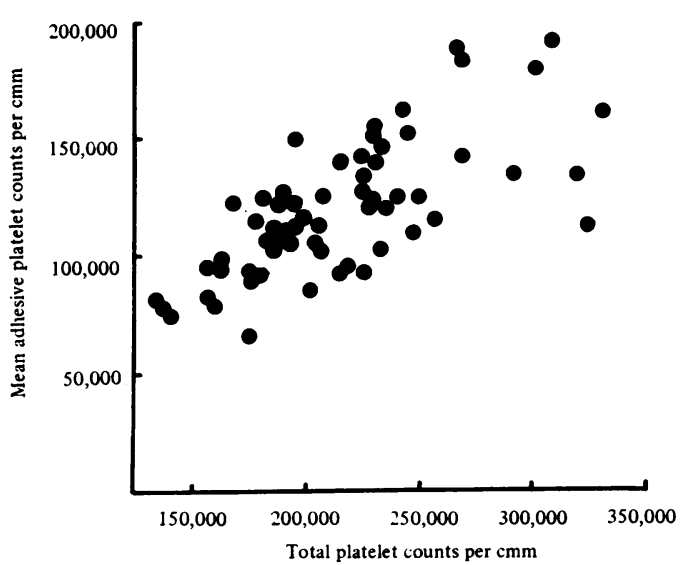

Fig. 1 The mean adhesive platelet count plotted against the corresponding mean total platelet count in six male volunteers and in 57 patients treated with long-term oral anticoagulants.

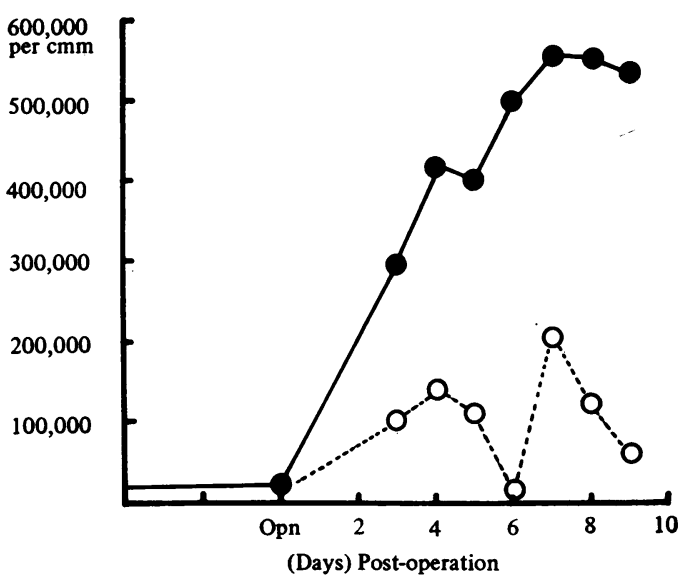

Fig. 3 Total platelet counts (solid circles) and ADPinduced adhesive platelet counts (open circles) in a 24-year-old woman with idiopathic thrombocytopenia treated by splenectomy.

total platelet count and the mean adhesive platelet count in the 63 subjects (Fig. 1). When the highest and lowest adhesive platelet counts found in each subject are plotted against the corresponding mean total platelet count (Fig. 2), it can be seen that most of the maximum values fall within 60 to $80 \%$, and most of the minimum values fall within 20 to $40 \%$ of the corresponding total platelet counts, the results showing a regular distribution (Eastham, 1966). No clinical condition was found to account for either very high or very low adhesive platelet counts. As reported

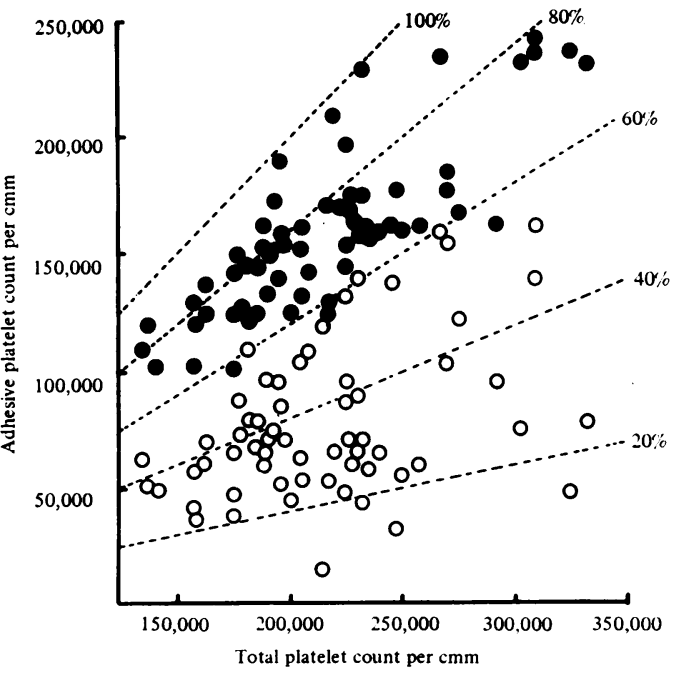

Fig. 2 The highest and lowest adhesive platelet counts plotted against the corresponding mean total platelet count in six male volunteers and in 57 patients treated with long-term oral anticoagulants.

previously (Eastham, 1966), the total platelet count in each case varied over the period of study by an average of \pm 10 to $15 \%$, and the corres-O ponding adhesive platelet count varied by an $\frac{0}{\varnothing}$ average of \pm 25 to $30 \%$.

Results obtained from the 24-year-old woman $\vec{O}$ treated by splenectomy for idiopathic thrombo-3 cytopenic purpura are shown in Figure 3. Before splenectomy the whole blood platelet count was persistently below 15,000 per $\mathrm{cmm}$. After splenec-중 tomy the whole blood platelet count rose during: the first seven days to exceed 500,000 per $\mathrm{cmm}^{3}$. and during this period the adhesive platelet count also increased but fluctuated markedly. By the eleventh day after operation clinical signs of pulmonary embolism were obvious with clinically? detectable venous thrombosis in either leg.

\section{Discussion}

Evidence that platelets play an important part in the development of atherosclerosis and arteriale

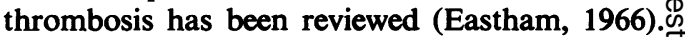
Unfortunately no useful prognostic value was obtained from total or adhesive platelet countso during long-term oral anticoagulant therapy (Eastham, 1966; Eastham and Avis, 1966). In an individual the total platelet count has been shown to vary about a mean value by an average of $10^{2}$ to $15 \%$ and the corresponding ADP-induced adhesive platelet count to vary by an average of 25 to $30 \%$ (Eastham, 1966). (It is obvious thato. the adhesive platelet count cannot exceed $100 \%$.) Re-examination of the latter results shows that 
there is a direct correlation between the mean adhesive platelet count and the corresponding mean total platelet count (Fig. 1). Since certain conditions are known to cause temporary thrombocytosis, for example, trauma, surgery, acute infection, venous and arterial thrombosis, and acute haemorrhage, it follows that a report of the adhesive platelet count should be expressed with the total platelet count, so that at some time it will be possible to decide whether the total platelet count is within normal limits for that particular subject.

The wide range of adhesive platelet counts (Fig. 2) without corresponding clinical changes in the patients studied or in the normal volunteers, and without obvious prognostic value in the prediction of future attacks of thrombosis, demonstrates the lack of clinical value of this estimation in patients with thrombotic lesions. The estimation of adhesive platelets in the method used in this study (Eastham, 1964) involves the addition of optimal amounts of ADP to heparinized blood, the ADP-induced adhesive platelets being removed by adhesion to a polystyrene surface, and the remaining non-adhesive platelets in the blood sample being counted. Subtracting the latter count from the total platelet count represents the ADP-induced adhesive platelets. The action of ADP on human platelets is not fully understood, but this substance causes a marked temporary increase in platelet adhesiveness. Measuring maximal production of platelet aggregation with ADP, Born (1966) has estimated that there must be about $2 \times 10^{5}$ ADP-binding sites on each platelet. Using the measurement of electrophoretic mobility of human platelets, Hampton and Mitchell (1966) estimated that each platelet had $0.85 \times 10^{5} \mathrm{ADP}$-binding sites, which is of a similar order of magnitude. These findings would explain the use of optimal concentrations of added ADP in the method used for the estimation of adhesive platelets. At ADP concentrations of less than $0.5 / \mu \mathrm{g} / \mathrm{ml}$ blood the induced adhesiveness is submaximal, whereas at concentrations of 0.5 to $10.0 / \mu \mathrm{g} / \mathrm{ml}$ blood the effect on the adhesive platelet count is maximal.

Analysis of platelets which were adhesive compared with platelets which did not adhere to a glass bead column did not show any difference in adenosine triphosphate content, rate of oxygen consumption, or in platelet volume, and Melchinger and Nemernos (1967) suggested that adhesion of platelets to glass was accompanied by a burst of metabolic activity and that only certain platelets had this potential property. This property does not appear to be constant in a platelet. Rozenberg and Holmsen (1968) showed that after incubation with ADP platelets lose their ability to aggregate on addition of more ADP and they suggest that there is a balance at any time between platelets capable of aggregating and platelets refractory to ADP, with ADP-responsive platelets becoming refractory and vice versa.
There is evidence that platelet adhesiveness isc controlled by plasma factors, and Besterman $\cong$ et al (1967) were able to demonstrate a diurna $\overline{\bar{F}}$ variation in the adhesive platelet count, probablyo due to the normal diurnal variation in circulating. adrenaline and noradrenaline, without a corre- sponding variation in the total platelet count. $\overrightarrow{\vec{F}}$ (Catecholamines such as adrenaline and nor $-\overrightarrow{0}$ adrenaline release ADP from the platelet substance to the platelet surface.) Further evidencees of humoral control of platelet adhesiveness, $\mathbb{\nabla}$ either with added ADP or without added ADP is that platelet adhesiveness increases within fours hours of surgical operation, especially when the $\overrightarrow{0}$ method of estimation includes erythrocytes from the patient (Bennett, 1968), and it is of interest that platelet adhesiveness and red cell electrophoretic migration rates are directly correlated (Davies and Lloyd, 1967). Similarly plasmaw obtained from subjects after exercise or stress $s_{+}^{u}$ shows that these factors grossly enhance platelet? aggregation (Harrison, Emmons, and Mitchell,응 1967). Although ADP is released from platelets. paradoxically platelets also carry enzymes for thec elimination of circulating ADP and this platelet 'ADP-ase' system is enhanced by a heat-stable $\overrightarrow{0}$ plasma factor (Howe and Bosshardt, 1968). It also appears that a lysolecithin, released from low-density lipoprotein by a plasma lecithinase, $O$ is responsible for marked changes in platelets electrophoretic mobility in response to ADP in patients suffering from arterial disease (Bolton $\frac{\Phi}{\mathbb{Q}}$ et al, 1967).

The fluctuating rise in adhesive platelets which $\overrightarrow{0}$ accompanied the thrombocytosis following splenectomy for idiopathic thrombocytopenia (Fig. 3) could be explained by the presence of factors affecting the development of adhesiveness in platelets, with unpredictable numbers of such:platelets trapped in an unsuspected developing? deep vein thrombosis and in the subsequento pulmonary embolism. Following the demonstration of the development of a stage wheno platelets are refractory to ADP (Rozenberg and? Holmsen, 1968) it is suggested that the estimationo of adhesive platelets by any method could be inefficient in demonstrating the presence of factors causing increased platelet adhesiveness. N It is possible that it might be better to estimate ${ }_{\mathrm{W}}$ the zeta potential at a standard glass-plasmao interface, since this measurement would correlate with the zeta potential at the platelet surface, and hence with the platelet adhesiveness, which increases with decrease in the negative surface 0 charge and its electrophoretic mobility (Dawberö and Roberts, 1968). The relationship between fall $\odot$ in zeta potential and aggregation of particles in human plasma has been investigated by Gardner ${ }^{\circ}$ (1969) who demonstrated that the normal state is unstable with zeta potential in the order of magnitude of threshold agglomeration of erythrocytes. By avoiding direct measurements ono platelets in this way, more delicate estimations of 
factors liable to increase the risk of thrombosis may become possible.

The risk of thrombosis in a vessel with an arteriosclerotic plaque on its wall is impossible to assess, but certain theoretical calculations are possible. The number of platelets flowing in blood with a platelet count of 250,000 per $\mathrm{cmm}$, through a small coronary artery of $2 \mathrm{~mm}$ internal diameter at a rate of $40 \mathrm{~cm}$ per second, is approximately $3 \times 10^{8}$ platelets per second. It is therefore not surprising that platelet thrombi can develop rapidly on such a patch. On the other hand, the wide spread of values for adhesive platelets (Fig. 3) have not been found to be clinically useful, and it is not difficult to offer an explanation. With eddy currents swirling and branching in smaller arteries, the blood will tend to be fully mixed, and coaxial flow will probably not occur. The risk of impact of platelets on a plaque will therefore be proportional to the cube root of the platelet count. Assuming platelet counts of $15,600,125,000,422,000$, and $1,000,000$ per $\mathrm{cmm}$ the order of risk of impact would be 25:50:75:100 or $1: 2: 3: 4$ only. The absence of clinical correlation with adhesive counts ranging from 30,000 per $\mathrm{cmm}$ to 240,000 per $\mathrm{cmm}$ in this series corresponds to a risk ratio of only $1: 2$ at the greatest difference found, if this suggestion is valid.

The estimation of the adhesiveness of platelets is clinically of value in the detection and classification of bleeding conditions associated with defective platelets, such as thrombocytasthenia (Glanzmann's disease), thrombopathia, and von Willebrand's disease.

\section{References}

Bennett, P. N. (1968). Role of erythrocytes in the acute platelet response to operation. J. clin. Path., 21, 695-697.
Besterman, E., Myat, G., and Travadi, V. (1967). Diurnal variation $\Omega$ of platelet stickiness compared with effects produced by adrenaline. Brit. med. J., 1, 597-600.

Bolton, C. H., Hampton, J. R., and Mitchell, J. R. A. (1967) Nature of the transferable factor which causes abnormaF platelet behaviour in vascular disease. Lancet, 2, 1101-1 105.0

Born, G. V. R. (1965). Uptake of adenosine and of adenosine diphosphate by human blood platelets. Natire (Lond.) 206, 1121-1122.

Davies, D. F., and Lloyd, V. (1967). Relationship betweero platelet adhesiveness and plasma surface activity in coronary heart disease. Cardiovasc. Res., 1, 108-110.

Dawber, J. G., and Roberts, J. C. (1968). An electrical double $e^{\bar{\Omega}}$ layer theory for platelet adhesiveness and initiation of intravascular thrombosis. Thrombos. Diathes. haemorrh? (Stuttg.), 19, 451-458.

Eastham, R. D. (1963). Rapid whole-blood platelet counting using an electronic particle counter. J. clin. Path., $16 \vec{\circ}$ 168-169.

Eastham, R. D. (1964). Rapid adhesive platelet count in whole $\vec{\omega}$ blood. J. clin. Path., 17, 45-46.

Eastham, R. D. (1966). Adhesive platelets and myocardia? infarction. Geriatrics, 21(7), 182-192.

Eastham, R. D., and Avis, P. R. D. (1966). Seasonal fluctuation in adhesive platelets during long-term anticoagulantw therapy. Brit. J. Haemat., 12, 39-43.

Gardner, B. (1969). Studies of the zeta potential of cells and silica particle in varying concentrations of albumin, $\mathrm{O}$ calcium, sodium, plasma and bile. J. Lab. clin. Med., 73, 202-209.

Hampton, J. R., and Mitchell, J. R. A. (1966). An estimate of the number of adenosine diphosphate binding sites on human platelets. Nature (Lond.), 211, 245-246.

Harrison, M. J. G., Emmons, P. R., and Mitchell, J. R. A. (1967) The variability of human platelet aggregation. J. Athero scler. Res., 7, 197-205.

Howe, E. E., and Bosshardt, D. K. (1968). A study of factors influencing the enzymatic elimination of adenosine diphosphate in blood. Thrombos. Diathes. heamorrh. (Stuttg.) 20,377-383.

Melchinger, D., and Nemerson, Y. (1967). Randomness of platelet adhesiveness with respect to their ATP, $\mathrm{O}_{2}$ consumption and size. J. appl. Physiol., 22, 197-200.

Negus, D., Pinto, D. J., and Brown, N. (1969). Platelet adhesive-응 ness in postoperative deep-vein thrombosis. Lancet, 1,0 220-224.

Pegrum, G. D., Shaw, S., and Wolff, S. E. (1967). Measuremenê of platelet adhesiveness including the use of diatomaceoussilica (celite). J. clin. Path., 20, 65-69.

Rozenberg, M. C., and Holmsen, H. (1968). Adenosine nucleotide metabolism of blood platelets. IV. Platelet aggregation response to exogenous ATP and ADP. Biochim. biophys Acta (Amst.), 157, 280-288. 